\title{
CONSISTENT SELF-SIMILAR MAGNETOHYDRODYNAMICS EVOLUTION OF CORONAL TRANSIENTS
}

\author{
David Shapakidze ${ }^{1}$, Arnold Debosscher ${ }^{2}$, Andria Rogava ${ }^{1,2,3}$, And StefaAn Poedts ${ }^{2}$ \\ ${ }^{1}$ E. Kharadze Georgian National Astrophysical Observatory, Ilia Chavchavadze State University, Tbilisi, Georgia \\ ${ }^{2}$ Centre for Plasma Astrophysics, Katholieke Universiteit Leuven, Leuven, Belgium \\ 3 Abdus Salam International Centre for Theoretical Physics, Trieste, Italy \\ Received 2009 March 10; accepted 2009 November 26; published 2010 March 4
}

\begin{abstract}
The self-similar model of coronal transients by B. C. Low is reconsidered. Due to a modification of the basic set of the initial assumptions of the model, a new class of more consistent solutions is found. The main advantage of these new solutions is that they do not contain areas with a physically inconsistent negative pressure. Instead, the novel solutions are derived on the basis of a special prescription for the thermal pressure of the transients that guarantees, by design, its positiveness throughout the whole evolution domain. The possible importance of these solutions for understanding the physics of the transient interplanetary coronal mass ejections (ICMEs; originating from the Sun), and magnetic clouds as a subclass of these, is discussed. A practical example is cited illustrating the application of our analytic results to describe some properties of real ICMEs. Some directions and scopes for further research are outlined.
\end{abstract}

Key words: methods: analytical - magnetohydrodynamics (MHD) - plasmas - solar wind - Sun: coronal mass ejections (CMEs)

\section{INTRODUCTION}

In almost all cases of astrophysical interest, the dynamics of plasma flows is governed by the combined influence of gravitational and electromagnetic fields. The difficulty of an accurate description of the large-scale motion of these flows, arising due to the dynamic influence of both these fields, is often quite unavoidable and generic, related to the complexity of involved field symmetries and the presence of multi-scale motions of the medium. Moreover, the difficulty of the study dramatically increases for higher dimensional and relativistic systems. But even for geometrically simplified, non-relativistic models the resulting equilibria are quite complicated, often defying any exact, analytic treatment.

Fortunately, in theoretical physics there are a number of methods that allow us to surmount problems of this kind by applying various simplifying phenomenological models, capturing main symmetries and kinematic features of the plasma dynamics. The application of these analytic methods drastically simplifies the mathematical aspect of the problems, up to the extent that they become analytically manageable and tractable. In a number of different applications, in this vein, it was proven that relatively consistent and reliable, approximate analytic results can be obtained. This becomes feasible if one deals with scale-invariant physical phenomena distinctive by their automorphism and the self-similarity of their geometry and dynamics.

In particular, from classic hydrodynamics, we know that the self-similar approach (hereafter referred to as SSA) has the advantage of simplifying time-dependent, multi-dimensional problems of intricate geometric and kinematic complexity, making them analytically tractable (Low 1990). The surprising efficiency of the SSA is related to the essential fact that a wide variety of dynamical systems allow us to establish the functional relation (if correctly guessed and found out) between two or more-temporal and spatial-variables, and thus to reduce the initial set of partial differential equations. In other words, the SSA enables us to describe the evolution of the reduced system, i.e., the simplified and analytically solvable problem, governed by a reduced set of ordinary differential equations in terms of functions of just one "master" variable, referred to as the selfsimilar variable.

This method has been fruitfully used in hydrodynamics (HD; Sedov 1946; Taylor 1950; Korobeinikov 1956; Barenblatt \& Zel'dovich 1972) for a long time. It was also well employed in a number of important magnetohydrodynamic (MHD), applications (Bernstein \& Kulsrud 1965; Kulsrud et al. 1965) helping to derive some interesting self-similar solutions for timedependent MHD flows without gravity. The application of the SSA to the MHD system in the presence of gravity is a much more challenging task, however. Still, there are several good examples of such results in plasma astrophysics (Tsinganos 1981; Trussoni \& Tsinganos 1993; Trussoni et al. 1997; Finn et al. 2004).

A remarkable series of self-similar MHD studies in the presence of gravity was initiated by B.C. Low in a number of his publications in the 1980s and 1990s where he has derived a class of self-similar solutions of the full set of ideal MHD equations for a $\gamma=4 / 3$ polytrope. In particular, he has studied coronal transients as $\gamma=4 / 3$ polytropes (Low 1982a), the expansion of a stellar envelope in a surrounding vacuum (Low 1982b), and different classes of spherically symmetric gasdynamic flows (Low 1984a). Low has also explored in more depth the physics of coronal transients (Low 1984b) and derived self-similar solutions for gravitating spheres and spheroids (Low 1992). Later, a similar approach was used for the three-dimensional, time-dependent CME model developed by Gibson \& Low (1998). The application of this theory is focused to mimic the configuration and the time-dependent evolution of the magnetized CMEs as revealed from observations (Dulk et al. 1976; Gosling et al. 1976; MacQueen 1980; Wagner et al. 1981; MacQueen \& Fisher 1983). The observations indicate the coherent nature of the geometry of large-scale magnetic structures of the moving transients, justifying the description of CMEs in terms of self-similar MHD.

Coronal transients (CTs) are eruptions of large amounts of outward propagating magnetized plasma in the solar corona. They are often observed as loop-shaped, white-light objects 
having sizes comparable to the solar radius or even larger and traveling with speeds of the order of a few hundred kilometers per second into the interplanetary medium (Low 1990). CMEs are the most energetic of the CTs, representing the sudden eruption of several billions of tons of overheated plasma. CMEs are recognized as the major cause of the largest and most hazardous space weather disturbances (Low 1990; Gosling 1993; Klimchuk 2001). Most of the principal issues related to the theory of CMEs - their initiation, evolution, and propagation, their structure, shock formation, and their relation to associated solar energetic particles-are not well understood and up to today remain in the list of most challenging problems of solar physics.

The main objective of this paper is to make a step forward in constructing reliable mathematical models describing the form and evolution of the CMEs. In this study, we largely follow the spirit of Low's innovative approach (Low 1982a), and we derive and describe a new class of self-similar CME models with positively prescribed pressure (or density). Essentially, our approach consists of Low's formalism, but it is adjusted or modified in order to ensure the physically necessary positiveness of the CMEs thermodynamic variables throughout the whole domain. This modification is by no means trivial, because Low's original solutions exhibited the pressure sign change behavior in certain limited parts of the domain occupied by the CMEs. Building on the positive pressure prescription, we derive new self-similar analytic solutions for the CMEs, and we analyze and discuss them.

The physically well-defined nature of our new class of solutions all the way up to infinity becomes decisive if one studies shock wave propagation accompanied by eruptive catastrophic events such as CMEs. The point is that in these cases it is of essential importance to work with physically meaningful shockconsistent solutions till infinity. Only under this condition can one accept the solution and admit that the approach is selfconsistent and adequately describe the CMEs, the related shock propagation and their evolution into the interplanetary space.

The organization of the paper is as follows. In Section 2, we manifest the main assumptions and basic set of equations of Low's SSA formalism and derive the new class of selfsimilar MHD solutions based on positively prescribed pressure; a practical example of application of our analytic results to describe the properties of some type of real CMEs is cited in Section 3; we discuss the possible advantages of our solutions for the physics of solar transients in Section 4; in the Appendix, we present the results of numerical analysis revealing some useful settings for physically meaningful solutions.

\section{SELF-SIMILAR MHD SOLUTIONS}

We will work with the complete set of ideal MHD equations, including gravity, as an external force. These equations can be written in the following form:

$$
\begin{gathered}
D_{t} \mathbf{v}=-\frac{1}{\rho}\left[\nabla p+\frac{1}{4 \pi} \mathbf{B} \times(\nabla \times \mathbf{B})\right]-\frac{G M}{r^{2}} \mathbf{e}_{r}, \\
D_{t} \rho+\rho \nabla \cdot \mathbf{v}=0, \\
D_{t}\left[\log \left(p / \rho^{\gamma}\right)\right]=0, \\
D_{t} \mathbf{B}=(\mathbf{B} \cdot \nabla) \mathbf{v}-\mathbf{B}(\nabla \cdot \mathbf{v}), \\
\nabla \cdot \mathbf{B}=0,
\end{gathered}
$$

where $\rho, p, \mathbf{v}$, and $\mathbf{B}$ denote the plasma density, thermal pressure, the velocity field, and the magnetic field, respectively, while $D_{t} \equiv \partial_{t}+(\mathbf{v} \cdot \nabla)$ stands for the convective time derivative. The symbol $G$ denotes the gravitational constant, $M$ is the solar mass, $r$ is the distance from the center of the Sun, while $\mathbf{e}_{r}$ is the radial unit vector. The MHD flow is supposed to be isentropic with the polytropic index $\gamma$ taken to be constant.

Low's SSA formalism (Low 1982a) is built on the basis of three assumptions, which can be formulated in the following way.

1. A self-similar variable. The temporal and the radial evolution of the system is governed by the combined self-similar variable:

$$
\zeta=r / \Phi(t)
$$

where $\Phi(t)$ is an arbitrary function of time that has to be specified.

2. Radial flow. It is assumed that the global plasma flow is radial:

$$
\mathbf{v}=v(r, \theta, t) \mathbf{e}_{r} .
$$

3. Axisymmetric magnetic field. In terms of spherical coordinates $(r, \theta, \varphi)$, the magnetic field is assumed to be axisymmetric, yielding that it is expressible in terms of two scalar functions $\tilde{A}$ and $\tilde{B}$ (Chandrasekhar 1952):

$$
\mathbf{B}=\frac{1}{r \sin \theta}\left[\frac{1}{r} \frac{\partial \tilde{A}}{\partial \theta} \mathbf{e}_{r}-\frac{\partial \tilde{A}}{\partial r} \mathbf{e}_{\theta}+\tilde{B} \mathbf{e}_{\varphi}\right] .
$$

The axial symmetry, i.e., $\partial_{\varphi}=0$, greatly simplifies mathematical treatment, while retaining, at the same time, the most important features of the coronal transient as a MHD phenomenon. Note that Equation (8) ensures that the Maxwell equation $\nabla \cdot \mathbf{B}=0$ is satisfied by design.

According to Low (1982a), the self-similar solution is surmised as a leading term in an asymptotic expansion of a nonself-similar solution, sufficiently far from the influence of the boundary and initial conditions. The latter conditions are different for any real coronal transient, depending on the variable physical circumstances leading to the eruption. Obviously, one can hardly expect these structures to be similar at the initial phase of their evolution. Instead, later on, self-similar solutions are supposed to represent the intrinsic evolutionary behavior of every individual CME as an evolving system. In other words, the idea is that sufficiently far from the place of its origin and sufficiently long after the peculiarities of its initiation, the evolution and the structure of a coronal transient ceases to depend on the random particularities of initial and boundary conditions: the "memory" about them is gradually "washed out" (Barenblatt \& Zel'dovich 1972).

As was found out by Low (1982a), these assumptions imply that the thermodynamic and magnetic variables in the $(\zeta, \theta)$ "space" acquire the following form:

$$
\begin{gathered}
\widetilde{A}(r, \theta, t)=\widetilde{A}(\zeta, \theta), \\
p(r, \theta, t)=\Phi^{-4} P(\zeta, \theta), \\
\rho(r, \theta, t)=\Phi^{-3} D(\zeta, \theta), \\
\widetilde{B}(r, \theta, t)=\Phi^{-1} Q(\widetilde{A}),
\end{gathered}
$$

while the $r$ and the $\theta$ components of the momentum equation reduce to

$$
\frac{\partial P(\zeta, \widetilde{A})}{\partial \zeta}+\left(\frac{G M}{\zeta^{2}}+\alpha \zeta\right) D(\zeta, \widetilde{A})=0
$$




$$
\begin{gathered}
\frac{\partial^{2} \widetilde{A}}{\partial \zeta^{2}}+\frac{\sin \theta}{\zeta^{2}} \frac{\partial}{\partial \theta}\left(\frac{1}{\sin \theta} \frac{\partial \widetilde{A}}{\partial \theta}\right)+Q(\widetilde{A}) \frac{d Q}{d \widetilde{A}} \\
+4 \pi \zeta^{2} \sin ^{2} \theta \frac{\partial P(\zeta, \widetilde{A})}{\partial \widetilde{A}}=0,
\end{gathered}
$$

where $\alpha$ is a constant. These are Equations (27) and (24), respectively, from Low (1982a).

\subsection{Positively Prescribed Pressure Solution}

Up to now, we were closely following Low's method and model, but at this point, we would like to introduce a new assumption and to find a qualitatively different class of selfsimilar solutions. In particular, in Low's model, an analytic ansatz for the scalar potential $\widetilde{A}$ of the magnetic field (Low $1982 b, 1984 a)$ has been used. This led to the derivation of selfsimilar solutions with a common property: in certain cases they exhibited a physically inconsistent negative pressure behavior. In the present study, our intention is to check whether the positiveness of the pressure, as a basic physical principle, can be guaranteed by implementing it somehow into the very design of the self-similar model. If such a prescription works, it would naturally replace the $\widetilde{A}$-ansatz of Low's SSA, and would enable us to find a new class of self-similar solutions where the positiveness of the thermal pressure would be guaranteed from the start.

Therefore, we prescribe the simplest positively defined form of the second-order polynomial for the pressure

$$
P(\zeta, \widetilde{A})=p_{2}(\zeta) \widetilde{A}^{2}+p_{1}(\zeta) \widetilde{A}+p_{0}(\zeta) \geqslant 0,
$$

where $p_{2}(\zeta), p_{1}(\zeta)$, and $p_{0}(\zeta)$ are the functions of the selfsimilar variable $\zeta$. For the pressure to be positively defined, it is sufficient to have

$$
p_{2}(\zeta)>0 \quad \text { and } \quad p_{0}(\zeta)>p_{1}^{2}(\zeta) / 4 p_{2}(\zeta)
$$

As regards the functional form $Q(\widetilde{A})$, it can still be freely prescribed, concretizing the form of the toroidal component of the magnetic field $B_{\varphi}$ (Low 1984b). We will consider the case when the term $Q(d Q / d \widetilde{A})$ is linear in $\widetilde{A}$. For this to happen, $Q(\widetilde{A})$ has to have the following form:

$$
Q^{2}(\widetilde{A})=b_{2} \widetilde{A}^{2}+b_{1} \tilde{A}+b_{0},
$$

where $b_{2}, b_{1}$, and $b_{0}$ have to be chosen for ensuring the positiveness of this function.

The substitution of the prescribed functions (15) and (17) reduces the elliptic partial differential Equation (14) to the following linear form:

$$
\zeta^{2} \frac{\partial^{2} \widetilde{A}}{\partial \zeta^{2}}+\sin \theta \frac{\partial}{\partial \theta}\left(\frac{1}{\sin \theta} \frac{\partial \widetilde{A}}{\partial \theta}\right)+F_{2}(\zeta, \theta) \widetilde{A}+F_{1}(\zeta, \theta)=0 .
$$

Here the following notations are used:

$$
\begin{gathered}
F_{1}(\zeta, \theta) \equiv p_{1}(\zeta) 4 \pi \zeta^{4} \sin ^{2} \theta+\left(b_{1} / 2\right) \zeta^{2}, \\
F_{2}(\zeta, \theta) \equiv p_{2}(\zeta) 8 \pi \zeta^{4} \sin ^{2} \theta+b_{2} \zeta^{2} .
\end{gathered}
$$

We further simplify Equation (18) by setting

$$
b_{1}=p_{1}=0\left(F_{1}=0\right)
$$

and reducing it to a homogeneous equation. Obviously, in doing so, we seriously crunch the possible range of functions $(15)$ and (17). In particular, Equation (21) means that both $P(\zeta, \widetilde{A})$ and $Q^{2}(\widetilde{A})$ are assumed to be monotonously increasing functions of $\widetilde{A}$.

The resulting homogeneous equation can be solved by the usual method of separation of variables. If one requires

$$
\sigma \equiv p_{2}(\zeta) 8 \pi \zeta^{4}
$$

to be a $\zeta$-independent arbitrary parameter in $F_{2}$, and one writes $\widetilde{A}$ as

$$
\widetilde{A}(\zeta, \theta) \equiv A(\zeta) T(\theta),
$$

then the original Equation (18) breaks into the following set of independent second-order ordinary differential equations:

$$
\frac{d^{2} A}{d \zeta^{2}}+\left(b_{2} \pm \frac{\lambda^{2}}{\zeta^{2}}\right) A=0
$$

and

$$
\frac{d^{2} T}{d y^{2}}+\left(\sigma \mp \frac{\lambda^{2}}{1-y^{2}}\right) T=0
$$

Here, a new variable $y \equiv \cos \theta$ has been introduced and $\lambda^{2}$ is the separation constant. We see that the mathematical side of the problem is reduced to the inspection of the set of Equations (24) and (25). Solving these equations for $T(y)$ and $A(\zeta)$, and plugging the results back into Equation (23), the solution for the scalar potential $\widetilde{A}(\zeta, \theta)$ of the magnetic field can be recovered.

\subsection{The Latitudinal Distribution of Scalar Potential, $T(\theta)$}

Let us study Equation (25) and look for the solution of $T(y)$, describing the latitudinal behavior of the $\widetilde{A}$. It can be easily rewritten as the stationary state Schrödinger-type equation:

$$
\frac{d^{2} T}{d y^{2}}+[\sigma-U(y)] T=0,
$$

where $U(y) \equiv \lambda^{2} /\left(1-y^{2}\right)$ is an effective potential and $\sigma$ stands for the eigenvalue.

Borrowing the terminology from quantum mechanics, we can say that the "potential well" $U(y)$ has "walls" of infinite height at $y= \pm 1($ or $\theta=0, \pi)$. Therefore, the "wave function" $T(y)$ has to tend to zero at these points (Landau \& Lifshitz 1981):

$$
\lim _{\theta \rightarrow 0, \pi} T(\theta) \equiv \lim _{y \rightarrow \pm 1} T(y)=0
$$

and this is equivalent to the following boundary condition for the latitudinal component of dipolar magnetic field, $B_{\theta}$ :

$$
\lim _{\theta \rightarrow 0, \pi} B_{\theta}=0
$$

implying, in turn, that the magnetic field solution has poles at $\theta=0, \pi$. This is the simplest and physically most reasonable configuration for the axi-symmetric magnetic field of a coronal transient.

If we search for the solution of Equation (26) in terms of power series

$$
T(y)=\sum_{n=0}^{\infty} \tau_{n} y^{n}
$$


this yields to the following three-term recurrence formula for the coefficients $\tau_{n}$ (Debosscher 1991):

$$
(n+2)(n+1) \tau_{n+2}+\left[\sigma-\lambda^{2}-n(n-1)\right] \tau_{n}-\sigma \tau_{n-2}=0 .
$$

Therefore, coefficients $\tau_{n}$ have the functional form of $\tau_{n}=$ $\tau_{n}\left(\sigma, \lambda^{2}\right)$, where $\sigma$ is the setting parameter of pressure (see Equation (22)) and $\lambda^{2}$ is the separation variable. Since $\tau$ 's with negative indices equal zero, for finding a solution it is sufficient to prescribe arbitrary constant values to $\tau_{0}$ and $\tau_{1}$ and to solve the system of Equations (30). Further, the substitution of the $\tau$ 's into the boundary condition (27),

$$
\lim _{y \rightarrow \pm 1} T(y)=\sum_{n=0}^{\infty} \tau_{n}\left(\sigma, \lambda^{2}\right)=0,
$$

leads to the derivation of the discrete spectrum of $\left(\sigma, \lambda^{2}\right)$ pairs related to the special solution of the problem (see the Appendix and figures therein).

\subsection{The Radial and Temporal Distribution of the Scalar Potential, $A(\zeta)$}

Next, we have to specify the radial and the temporal evolution of $\widetilde{A}$, governed by the solution of Equation (24). The selection of the "-" sign in Equation (25) leads to the selection of the "+" sign in Equation (24). In this case, it comprises the Bessel-type equation

$$
\frac{d^{2} A(\zeta)}{d \zeta^{2}}+\left[b_{2}-\frac{\left(\mu^{2}-1 / 4\right)}{\zeta^{2}}\right] A(\zeta)=0
$$

where $\mu^{2} \equiv\left(1 / 4-\lambda^{2}\right)$ (Abramovitz \& Stegan 1972). Solutions of Equation (32) can be written either in terms of regular Bessel functions or in terms of the modified Bessel functions, depending on whether the parameter $b_{2}$ is positive or negative, respectively. The regular Bessel functions are characterized by an oscillating behavior along the $\zeta$-axis. Therefore, they specify magnetic fields with a so-called "multi-blob" structure along the radial direction. They could describe processes within some limited areas, tracing the evolution of individual "blobs" (Low 1984b, 1992), but they cannot describe the solar transients as a whole from the solar surface up to larger radii.

Here, however, we focus our attention rather on the second branch of solutions of Equation (32), specified by negative values of the parameter $b_{2}$. These solutions are given by modified Bessel functions of the second kind, $K_{\mu}$, in the form of

$$
£_{\mu}(b \zeta)=e^{i \mu \pi} K_{\mu}(b \zeta)
$$

where $-b^{2} \equiv b_{2}$. The modified Bessel function of the second kind, $K_{\mu}$, has the advantage to mimic a dipolar magnetic field throughout the entire area, while the modified Bessel function of the first kind, $I_{\mu}$ does not vanish at infinity. The $K_{\mu}$ are real functions, when $\mu>-1$, and tend to zero as $\zeta \rightarrow \infty$. Although $K_{\mu}(\zeta)$ are infinite at the origin, the special solution of Equation (32),

$$
A_{\mu}(\zeta)=a \sqrt{\zeta} £_{\mu}(b \zeta),
$$

has a maximum and is bounded from above (see Figure 1). Here $a$ is an arbitrary constant, and it serves as a scaling parameter for $A_{\mu}(\zeta)$.

A further detailed analysis shows that ascribing $\mu=0$ (yielding $\lambda^{2}=1 / 4$ ) results in a physically meaningful solution

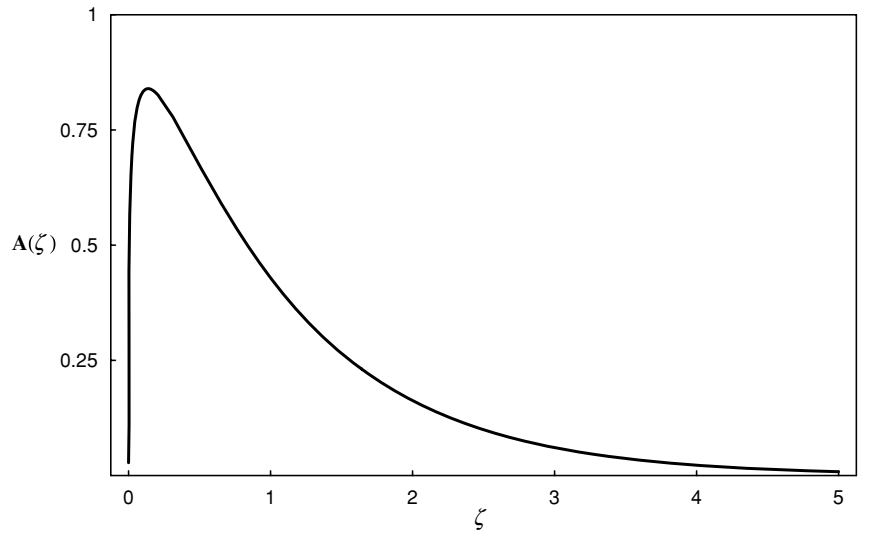

Figure 1. Profile $A(\zeta)$, normalized on the value $9.4 \times 10^{25}$ gauss $\mathrm{cm}^{2}$. It represents the special solution of Bessel-type Equation (32) in the region $\zeta \leqslant 5$, with $a=4 \times 10^{20}$ gauss $\mathrm{cm}^{3 / 2}, b=1.2 \times 10^{-11} \mathrm{~cm}^{-1}$, and $\mu=0 . A(\zeta)$ is the radial-temporal distribution of scalar potential $\widetilde{A}$ in terms of similarity variable $\zeta$ normalized on the value $R_{0} / \Phi\left(t_{0}\right)$, where $\Phi\left(t_{0}\right)=\eta^{1 / 2} t_{0}=2^{6 / 7}$, $R_{0}=10^{11} \mathrm{~cm}$, and $t_{0}=3686 \mathrm{~s}$.

for $\widetilde{A}(\zeta, \theta)$. Due to the reasonings discussed in the Appendix, borrowing again the terminology from quantum mechanics, the "ground state" solution is physically favorable for our model:

$$
\widetilde{A}(\zeta, \theta)=A_{\mu_{0}}(\zeta) \cdot T_{\sigma_{0}}(\theta)=a \sqrt{\zeta} £_{\mu_{0}}(b \zeta) \cdot T_{\sigma_{0}}(\theta)
$$

where $\mu_{0}= \pm \sqrt{1 / 4-\lambda_{0}^{2}}=0, \lambda_{0}^{2}=\lambda^{2}\left(\sigma_{0}\right)=1 / 4$, and $\lambda^{2}(\sigma)$ is the solution of Equation (31).

\subsection{The Self-similar Solution}

Finally, bearing in mind the definition (Equation (22)), from Equation (15) we can reconstruct the following expression for the pressure in terms of $r, \theta$, and $t$ :

$$
p(r, \theta, t)=\Phi^{-4} P(\zeta, \theta)=p_{A}(r, \theta, t)+p_{s}(r, t),
$$

where

$$
\begin{aligned}
p_{A}(r, \theta, t) & =\Phi^{-4}(t) \frac{\sigma}{8 \pi r^{4}} \widetilde{A}^{2}, \\
p_{s}(r, t) & =\Phi^{-4}(t) p_{0}(\zeta) .
\end{aligned}
$$

Accordingly, the expression for the density can be derived from Equation (13), considering the case of inertial flows, setting $\alpha=0$ (Low 1982a, 1984a, 1984b):

$$
\rho(r, \theta, t)=\Phi^{-3}(t) D(\zeta, \theta)=\rho_{A}(r, \theta, t)+\rho_{s}(r, t),
$$

where

$$
\begin{gathered}
\rho_{A}(r, \theta, t)=\Phi^{-3}(t) \frac{\sigma}{2 \pi G M} \frac{1}{r^{3}} \widetilde{A}^{2}, \\
\rho_{s}(r, t)=-\Phi^{-3}(t) \frac{\zeta^{2}}{G M} \frac{d p_{0}(\zeta)}{d \zeta} .
\end{gathered}
$$

The expressions for $p_{s}$ and $\rho_{s}$ are generated through arbitrarily defined form $p_{0}(\zeta)$ (see Equation (15)). It is easy to show that $p_{0}(\zeta)$ should be a positive function. Indeed, the quantum mechanical analogy ascertains that in the case of a "potential well" of the form of $U(y)=\lambda^{2} /\left(1-y^{2}\right)$, the "bounded state" solutions of Equation (26) correspond to the positive eigenvalues $E \sim \sigma>0$. Therefore, as far as $\sigma$ is related to $p_{2}(\zeta)$ via Equation (22), $p_{2}(\zeta)>0$. Then, taking into account the setting 

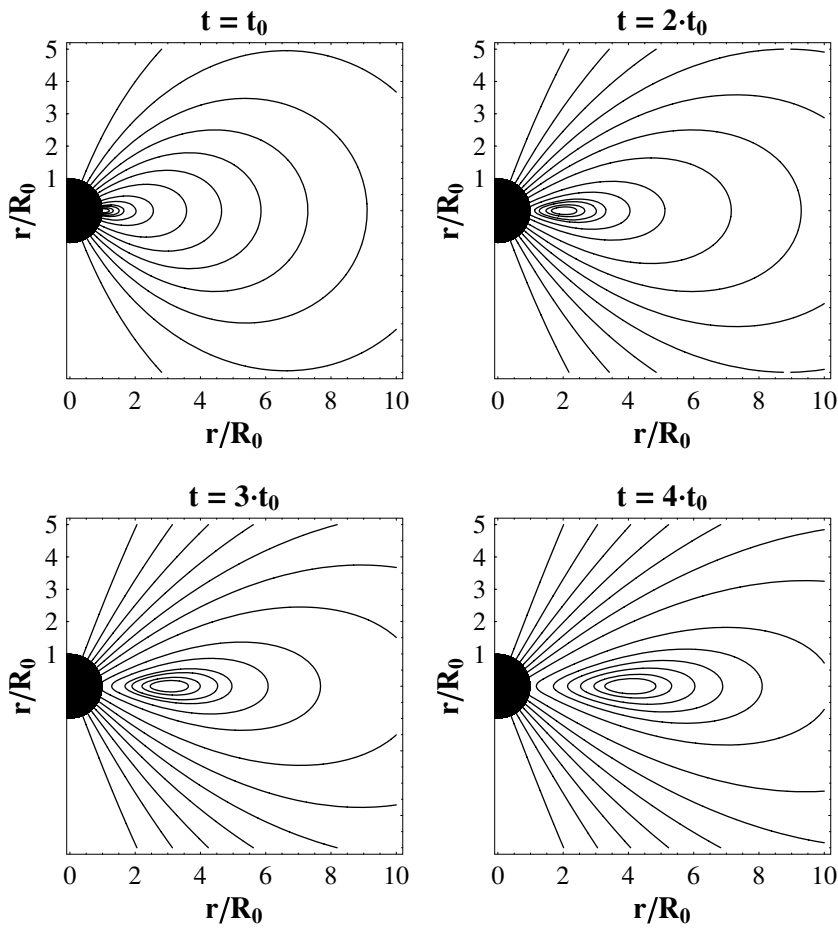

Figure 2. Flux-rope topology in the magnetic field. Evolution of the magnetic field lines is given in the $(r, \theta)$ plane by contours of constant $\widetilde{A} /\left(B_{0} R_{0}^{2}\right)$, where $B_{0}=1$ gauss and $R_{0}=10^{11} \mathrm{~cm}$. The nested set of closed lines belongs to a twisted toroidal flux rope at four different times $\left(t_{0}, 2 t_{0}, 3 t_{0}\right.$, $4 t_{0}$, where $\left.t_{0}=3689 \mathrm{~s}\right)$ during its progress from the Sun $\left(r=R_{0}\right)$ to $10 R_{0}$. Computations are done for the following settings: $a=2 \times 10^{20}$ gauss cm $\mathrm{cm}^{3 / 2}$ and $b=3 \times 10^{-12} \mathrm{~cm}^{-1}$ which are in accordance with the total pressure magnitude considered in Section 3 .

$p_{1}=0$ in Equation (19), our polynomial form for the pressure (see Equation (15)) is positively defined if $p_{0}(\zeta)$ is the positive function.

Regarding the free parameters appearing in the expression for the toroidal field $Q$, they are defined from the requirement of $Q^{2} \gtrsim 0$ in Equation (17). Taking into account that $b_{1}=0$ and $b_{2}=-b^{2}$, we see that $b_{0}$ and $b$ have to define upper and lower limits for the function $\widetilde{A},-\left(\sqrt{b_{0}} / b\right) \leqslant \widetilde{A} \leqslant\left(\sqrt{b_{0}} / b\right)$, in order to ensure $Q^{2} \gtrsim 0$. Eventually, the free parameter $b$ controls the form of spatial and temporal variation of $\widetilde{A}$, while its concrete value is determined by another free parameter, $a$ in Equation (34). The free parameter $b_{0}$ defines the value of the self-similar toroidal component of the magnetic field, $Q$. The manifested freedom in the choice of parameters makes the approach flexible enough and helps us to emulate the desired magnitude and morphology of the magnetic field in the domain of interest.

Numerous authors indicate that the geometry of magnetic field of CME's are consistent with, so-called, flux-rope morphology (see, e.g., Forbes et al. 2006 and references therein). Many CME's have what is commonly known as a dark cavity underneath, located between a bright frontal loop and an embedded bright core. It is suggested that such cavities correspond to magnetically highly energized flux-ropes upwardly streaming together with CME flow through the interplanetary space (Klimchuk 2001). The flux-rope topology is very different. However, in the most cases, field lines form a helical structure that is disconnected from the photosphere and moves outward the solar surface.

Here we demonstrate how well the model of our magnetic field topology is in accordance with the flux-rope topology of the CME's magnetic field. In Figure 2, we illustrate the twodimensional plot of the temporal evolution of the magnetic field vector potential (see Equation (35)) that is the solution of Equation (18). The field lines are drawn projected on the $(r, \theta)$ plane. A nonzero component should be kept in mind when visualizing this axisymmetric magnetic field in threedimensional space. From this panel, it is evident that the contours of the vector potential, $\widetilde{A}$, resembles the dipolar magnetic field with a moving loop structure along the equator. The loop is similar to the nested set of closed lines embedded in a dipole-like field that was shown in Figure 5 of Low (1984b). We suppose, that the both structures (loops in Figure 2 and nested set of closed field lines in Low 1984b) belong to a twisted toroidal flux-rope that runs around the Sun in the equatorial region. The similarity between the flux-rope topology and the panel in Figure 2 leads us to the assumption, that our model, at least, does not contradict the existence of the flux-rope configurations in the topology of CME's magnetic field. The computations are done for the following settings of free parameters in solution (35): $a=2 \times 10^{20}$ gauss cm $\mathrm{cm}^{3 / 2}$ and $b=3 \times 10^{-12} \mathrm{~cm}^{-1}$ which are in accordance with the total pressure magnitude considered below, in Section 3 of the present paper. We hope that in the next stage of corresponding precise tuning of free parameters will make the model applicable for the full description of particular CME event.

\subsection{The Shock Consistent Background Wind}

The self-similar, pressure-prescribed solutions, given by Equations (35)-(41), describe the axisymmetric, radially streaming global MHD flow. In order to make this analytic solution feasible for the problem of the coronal transients, one has to consider the shock-consistent solutions. For instance, the model for the coronal transients, described by Low (1984b), suggests an expulsive flow plowing into the preexisting ambient atmosphere. This arrangement results in a moving contact surface (CS) and an outward propagating shock front (SF). The analysis is based on the assumption that the self-similar MHD flow can be superimposed on a gasdynamic shock driving spherically symmetric background wind. In our case, the shock driving background wind is constructed by means of the arbitrary functions $p_{s}(r, t)$ and $\rho_{s}(r, t)$. The latter are prescribed by the hydrodynamic shock-consistent solutions for non-magnetized spherically symmetric flows (Low 1984a). These functions can describe the shock-consistent spherical flow similar to the dynamically consistent spherically symmetric atmosphere case for magnetic field prescribed solutions in paper by Low (1984b).

The complete analysis of the spherically symmetric hydrodynamic flow, expanding into a static ambient atmosphere, was presented by Low (1984a). Instead of repeating the arguments of this study, we here only present its final results in Table 1. It specifies the following spatial regions: (1) the static, nonmagnetized outflow behind the CS $\left(r<R_{c}\right)$; and the region of the ambient atmosphere $\left(r>R_{c}\right)$, which in turn, is split up by the SF and consists of (2) the post-shock, swept-up ambient medium $\left(R_{c}<r<R_{S}\right)$ and (3) yet undisturbed, the static pre-shock ambient atmosphere $\left(r>R_{s}\right)$.

In Figure 3, we show the total density profile of the flow which comprises our analytic solution, $\rho_{A}(r, t)$ (see Equation (40)) superimposed on the density of spherically symmetric shockconsistent flow (see $\rho_{s}$ in Table 1) derived by Low (1984a). Figure 3 illustrates that the contribution of gasdynamic spherical flow $\left(\rho_{s}\right)$ dominates over the magnetized flow $\left(\rho_{A}\right)$ in the selfsimilar solution. Therefore, we can neglect the influence of 
Table 1

Data for the Spherically Symmetric Radial Flow

\begin{tabular}{|c|c|}
\hline & $r=R_{0}:$ Solar Surface (SS) \\
\hline \multirow[t]{2}{*}{ (1) } & $\begin{array}{c}r \leqslant R_{c}(t): \text { outflow region } \\
p_{s}(\zeta)=p_{\text {out }}(r)=\frac{1}{4 v^{3}}\left(\frac{G M}{r}\right)^{4} \\
\rho_{s}(\zeta)=\rho_{\text {out }}(r)=\frac{1}{v^{3}}\left(\frac{G M}{r}\right)^{3} \\
\left(\frac{G M}{v R_{0}}\right)^{3}=10^{8} \cdot m_{p} \mathrm{~cm}^{-3} \\
R_{0}=10^{11} \mathrm{~cm} \\
m_{p}=\text { proton mass } \\
M=M_{\odot}\end{array}$ \\
\hline & $r=R_{c}(t):$ Contact Surface $(\mathrm{CS})$ \\
\hline \multirow[t]{8}{*}{ (2) } & $R_{c}(t) \leqslant r \leqslant R(t):$ post-shock ambient \\
\hline & $p_{s}(\zeta)=p_{\text {post }}(\zeta)=\frac{7}{6} \Phi^{-4} d_{0} \eta R_{0}^{2} \exp \left[\frac{2}{3} \frac{G M}{\eta R_{0}^{3}}\left(\frac{R_{0}}{\zeta}\right)^{9}\right]$ \\
\hline & $\rho_{s}(\zeta)=\rho_{\text {post }}(\zeta)=7 \Phi^{-3} d_{0}\left(\frac{R_{0}}{\zeta}\right)^{8} \exp \left[\frac{2}{3} \frac{G M}{\eta R_{0}^{3}}\left(\frac{R_{0}}{\zeta}\right)^{9}\right]$ \\
\hline & $d_{0}=10^{8} m_{p} \frac{6}{7} \frac{G M}{\eta R_{0}^{3}}\left(\frac{R_{0}}{\zeta_{c}}\right)^{18} \exp \left[-\frac{2}{3} \frac{G M}{\eta R_{0}^{3}}\left(\frac{R_{0}}{\zeta_{c}}\right)^{9}\right] \approx 7 \times 10^{6} m_{p} \mathrm{~cm}^{-3}$ \\
\hline & $\Phi\left(t_{0}\right)=\eta^{1 / 2} \cdot t_{0}=2^{6 / 7}$ \\
\hline & $\begin{array}{c}t_{0}=3686 \mathrm{~s} \\
\end{array}$ \\
\hline & $\zeta_{c}=2 R_{0} / \Phi\left(t_{0}\right)=2^{1 / 7} \cdot R_{0}$ \\
\hline & $r=R_{c}(t):$ Shock Front $(\mathrm{SF})$ \\
\hline \multirow[t]{2}{*}{ (3) } & $r \geqslant R(t):$ pre-shock ambient \\
\hline & $\rho_{s}(\zeta)=\rho_{\mathrm{amb}}(r)=d_{0} \cdot\left(\frac{R_{0}}{r}\right)^{26 / 7} \exp \left[\frac{2}{3} \frac{G M}{\eta R_{0}^{3}}\left(\frac{R_{0}}{r}\right)^{9 / 7}\right]$ \\
\hline
\end{tabular}

the magnetic field on the flow. Consequently, we can consider the shock formation in the hydrodynamic regime, which is in accordance with Low (1984a). In Figure 4, we plot the temporal evolution profile (the equatorial flow, $\theta=\pi / 2$ ) of the shockconsistent self-similar MHD solutions for total density and total pressure of the flow (see Equations (36)-(41)). The snapshots trace the shock region, included by CS and SF, for three different sequential instants of time. The figure illustrates the physically necessary positiveness of thermodynamic variables throughout the whole domain (the plots show the expansion of transient up to $10 R_{0}$, where $R_{0}$ is the solar radius).

\section{FITTING THE ANALYTIC MODEL TO CME OBSERVATIONS}

In this section, we make an attempt to show how well the results of our analytic model can be applied to study the properties of real interplanetary CMEs.

Not long ago, Jian et al. (2006) have presented a comprehensive survey of 230 interplanetary CMEs during 1995-2004 using observations near $1 \mathrm{AU}$. The compound parameter of the total pressure, $P_{t}$, assists authors in effectively distinguishing ICMEs from other solar wind structures and further classification of this phenomenon. Particularly, Jian et al. (2006) categorize ICMEs into three major groups specified by the characteristic signatures of $P_{t}$ temporal variation at $1 \mathrm{AU}$ from the solar surface. The temporal behavior of $P_{t}$ and other wind parameters (such as magnetic field components, solar wind bulk velocity magnitude, the proton number density, the proton temperature, and the ratio of plasma pressure to magnetic pressure) are all illustrated in three plot panels displayed as Figures $2-4$ in the paper by Jian et al. (2006). All three groups show the rapid rise in $P_{t}$ at the beginning of the profiles sometimes directly associated with the shock front. However, the events of Group 3 (see Figure 4 by Jian et al. 2006) are particularly characterized by their smoothly declined profile of a post-shock temporal evolution.

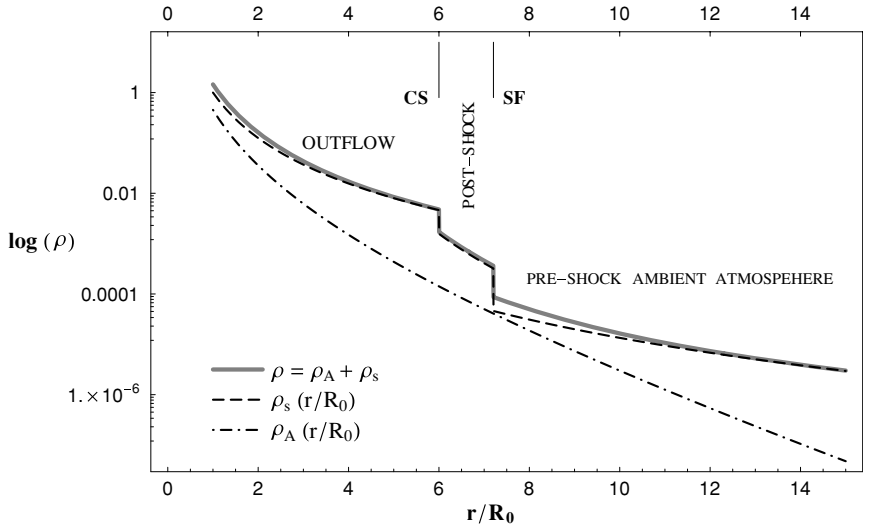

Figure 3. Total density profile of equatorial flow, $\theta=\pi / 2$. The figure illustrates that the contribution of gasdynamic spherical flow dominates over the magnetized flow in the self-similar solution. Positive-pressure-prescribed solution of MHD flow, $\rho_{A}$, (see Equation (40)) is superimposed on the hydrodynamic shock-consistent solution of spherical flow, $\rho_{s}$, given by Table 1. Computations are done with the following settings: $a=4 \times 10^{20} \mathrm{gauss} \mathrm{cm}^{3 / 2}$, $b=1.2 \times 10^{-11} \mathrm{~cm}^{-1}, \mu=0$, and $t=3 t_{0}$, where $t_{0}=3689 \mathrm{~s}$. The radial distance is measured in terms of the solar radii, $R_{0}$. The normalization is $\rho_{s}\left(R_{0}\right)=10^{8} m_{p} \mathrm{~cm}^{-3}$, where $m_{p}$ is a proton mass.

In our model, the smooth post-shock pressure decay becomes crucial, because the self-similar solutions are built up on modified Bessel functions of the second kind, $K_{\mu}$ (see Equations (33), (35) and (37)), depicting very similar profile behavior. Therefore, since Group 1 (G1) includes events that show the apparent enhanced $P_{t}$ after the shock in the middle of the temporal axis and ICMEs in Group 2 (G2) are characterized by post-shock pressure plateau and much later return to earlier lower pressure stage, we focus on Group 3 (G3) events which show aftershock gradual pressure decay lasting over dozens of hours or days. Our analytic self-similar solution for the pressure can easily be adjusted to such a profile: indeed, the prescription of proper values to the available free parameters, $a$ and $b$ in Equation (35), successfully fits the total pressure profile of analytic self-similar solution (see Equations (36)-(38)), with $P_{t}$ profile of G3 events derived from the observations (compare Figure 5 in our paper and $P_{t}$ profile in Figure 4 by Jian et al. 2006).

Jian et al. (2006) interpret the three groups of ICMEs as corresponding to different distances of the spacecraft passes through the ICME relative to the central flux rope. For example, they suggest that G3 events catch the shock away from the equatorial flow. In our model, the consideration of pressure profile of the equatorial flows $(\theta=\pi / 2$ in Figure 5) instead of poloidal flows, however, does not affect the profile shape behavior. The magnitude of the main wind parameters could always be properly scaled by the corresponding set of free parameters available in our solution.

The choice of G3 events, as a real CME example that can be described by our analytic results, is supported by their proton number density profile as well (see $N_{p}$ profile in Figure 4 by Jian et al. 2006). Its comparatively smooth, almost hyperbolic temporal decay makes G3 events suitable to our $K_{\mu}$-functionbased solutions (see Equations (33), (35) and (40)), whereas G1 events are prominent with irregularities in $N_{p}$ temporal evolution and G2 events maintain almost constant temporal distribution of $N_{p}$ in magnetosheath interval and nearly parabolic decline in the region of magnetic obstacle (see Figures $2-3$ by Jian et al. 2006).

Moreover, the observed wind bulk velocity profile in the panel of G3 events (see $V_{p}$ profile in Figure 4 by Jian et al. 

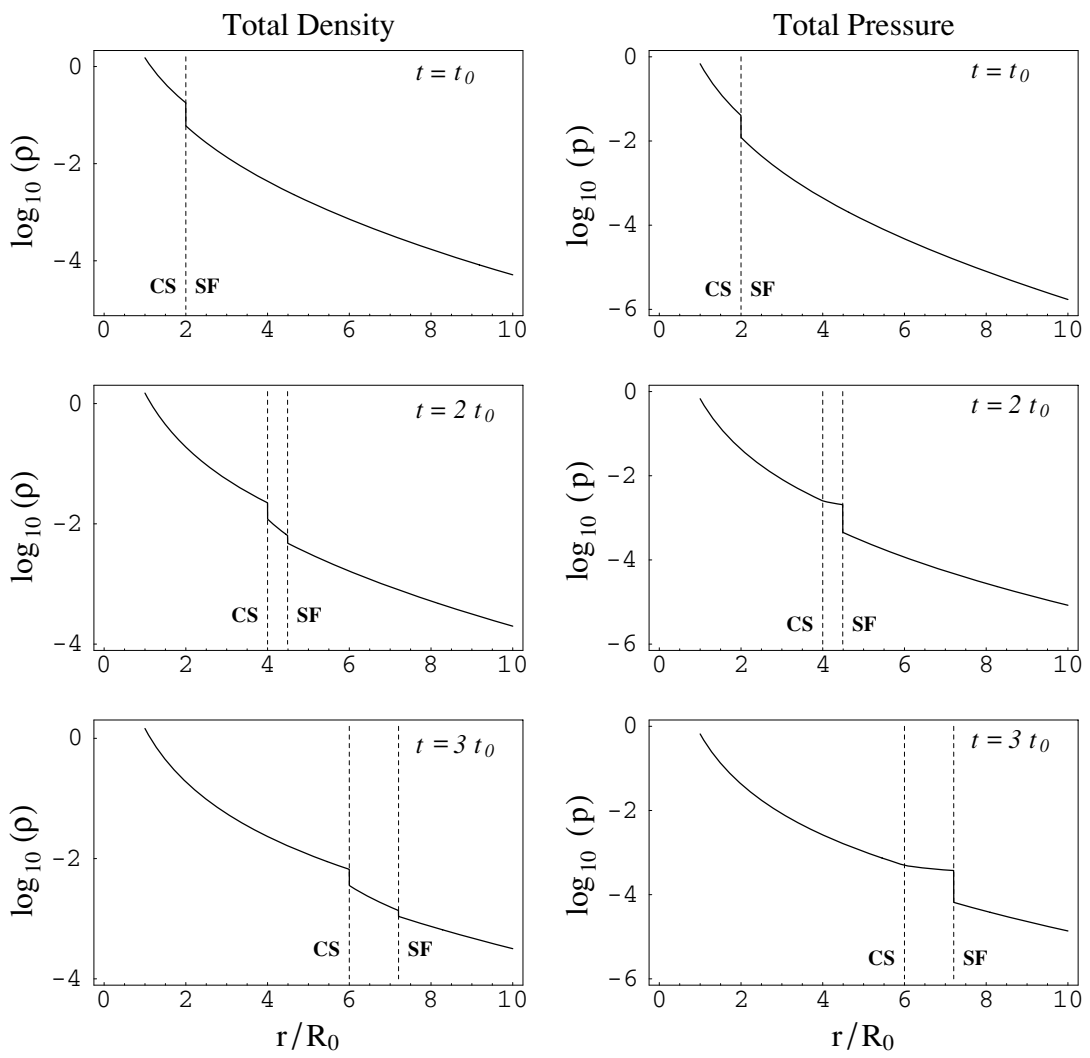

Figure 4. Temporal evolution of the pressure-prescribed self-similar solution (see Equations (39) and (36), respectively). The profiles of total pressure and total density of the equatorial flow, $\theta=\pi / 2$, are presented. Positive-pressure-prescribed solutions of MHD flow, $\rho_{A}$ and $p_{A}$, (see Equations (40) and (37), respectively) are superimposed on the hydrodynamic shock-consistent solutions of spherical flow, $\rho_{s}$ and $p_{s}$, given by Table 1 . The normalizations are $\rho_{s}\left(R_{0}\right)=10^{8} m_{p} \mathrm{~cm}^{-3}$ and $p_{s}\left(R_{0}\right)=\left(G M / 4 R_{0}\right) \times 10^{8} m_{p} \mathrm{~cm}^{-1} \mathrm{~s}^{-2}$ for density and pressure, respectively. $m_{p}$ is a proton mass. The scalar potential, $\widetilde{A}$, is calculated for the following settings: $a=4 \times 10^{20}$ gauss cm $\mathrm{cm}^{3 / 2}, b=1.2 \times 10^{-11} \mathrm{~cm}^{-1}$, and $\mu=0$. The snapshots trace the shock region, included by CS and SF, for three different sequential instants of time: $t_{0}, 2 t_{0}, 3 t_{0}$, where $t_{0}=3689 \mathrm{~s}$. The figure illustrates the physically necessary positiveness of thermodynamic variables throughout the whole domain. The radial distance is measured in terms of the solar radii, $R_{0}$.

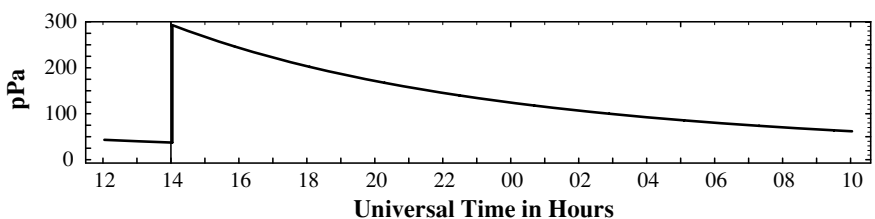

Figure 5. Analytic model of temporal evolution of the total pressure of equatorial flow $(\theta=\pi / 2)$ at $r=1$ AU from the solar surface. The plot represents a graph of the function, $p_{A}$, given by Equation (37) superimposed on the pressure solutions for a gasdynamic shock driving spherically symmetric background wind, $p_{s}$, listed in Table 1: $p(t)=\Phi^{-4}(t) \cdot\left[a \zeta^{1 / 2} K_{\mu}(b \zeta) \cdot T_{\sigma}(\theta)\right]^{2} \cdot \sigma /\left(8 \pi r^{4}\right)+p_{s}(t)$. The total pressure $\left(p \cdot 10^{11}\right)$ is measured in the unit of pico-Pascal $(\mathrm{pPa})$. As is argued in the Appendix, $\mu=0$, so $\sigma \approx 2.77$. The following settings are applied to free parameters: $a=2 \times 10^{20}$ gauss cm $\mathrm{cm}^{3 / 2}$ and $b=3 \times 10^{-12} \mathrm{~cm}^{-1}$. Universal time in hours is chosen for scale units along the temporal axis, analogous to scaling of the temporal axis in Figure 4 by Jian et al. (2006).

2006) demonstrates the notable coincidence with the assumption of inertial flows in our paper. The self-similar solution (Equation (40)), together with superimposed spherically symmetric background wind by Low (1984a), are both derived for inertial flows setting $\alpha=0$ in Equation (14). The post-shock deceleration/acceleration of the flow is poorly observable for G3 events (especially in the wide interval of magnetic obstacle), whereas G1 and G2 events are conspicuous by their post-shock decelerating speeds throughout the whole temporal axis of the panel (see $V_{p}$ profiles in Figures $2-3$ by Jian et al. 2006).

On the bases of this analysis, we suppose that G3 ICMEs indeed are suitable candidates for the description in the framework of our analytic self-similar, pressure-prescribed model.
We expect, therefore, that the different parameters of ICMEs, classified as G3 events, may be successfully estimated and predicted by tracing the evolution of spacial-temporal behavior of our analytic solutions.

\section{DISCUSSION AND CONCLUSIONS}

In the present paper, an exact analytic solution of the full set of MHD equations (superposed on shock-consistent solution of hydrodynamic equations) has been presented for a shock driving coronal transient MHD flow out of the gravitational bounds of the Sun. The problem is treated in the framework of the self-similar theory. The self-similarity is not only a method to make mathematics tractable, but it can also be considered as an intrinsic feature of the coronal transients. The observed large-scale structures of the coronal transients preserve their coherence during the outward motion from the Sun. This is justified from the images of white-light coronagraphs (Munro 1977) where the transients are revealed in the form of bright leading loops. CMEs belong to this type of transients. Observations of CMEs show an ordered expansion of the ejected matter propagating forward in the solar ambient atmosphere and led by the bright loop structure associated with the shock front (Burkepile \& Cyr 1993). Since CMEs maintain an impressive coherent form, this suggests describing their evolution by means of the self-similar theory (Gibson \& Low 1998).

Low's approach (Low 1982a) was based on a set of assumptions about self-similar behavior of the transients, their geometry, and the structure of their magnetic fields. On the basis of 
these assumptions, the exact self-similar solutions were derived. However, when shock is present in the flow, the "magnetic field prescribed" solutions (Low 1984b) appears physically acceptable only within a limited spatial-temporal area, initially located in the vicinity of the contact surface. In other areas, these solutions exhibit paradoxical and physically unjustified negative thermal pressure values. On the other hand, numerous observations indicate the coherent and self-similar appearance of the coronal transients within the broad area $2 R_{0}<r<5 R_{0}$ and over large intervals of time. Therefore, it seems quite interesting to find self-similar solutions that would retain physically valid behavior for longer time spans and over broader spatial range. In particular, it is important to have outward solution which do not contain density and pressure depletion cavities related with the negative values of these quantities, as it turns out to be the case of the third example in the paper by Low (1984b).

In order to study coronal transients and CMEs, the evolution of the entire shock region (including CS and SF) has to be specified. According to the solution by Low (1984b), the leading shock front as well as contact surface, upon their formation at $r=2 R_{0}$, leave the "favorable area" where the self-similar solution is physically meaningful. To avoid this discrepancy, in this paper we derived a new class of self-similar solutions that stay physically well-defined throughout the entire spatial-temporal domain. For this purpose, we introduce a different heuristic approach and change the basic set of Low's assumptions: instead of postulating the form of the magnetic field, we introduce a new ansatz for the thermal pressure that guarantees its positiveness throughout the whole area of interest. This is achieved by a mathematical prescription for the pressure variable in the form of a second-order polynomial. The positivepressure-prescribed solution allows, but not ensures, the rest of the physical variables (i.e., the density and the magnetic field) to remain physically meaningful throughout the whole domain.

Generally, if one requires a solution to be physical, then it should satisfy the appropriate set of initial/boundary conditions. Self-similar solutions satisfy initial/boundary conditions rigidly related to their own design. Only in favorable cases, when one has the freedom to modify the self-similar solutions by means of available free parameters, is it possible to adjust them to real initial/boundary conditions characterizing the real physical object or process. This is the case with our model: the special self-similar solution (Equation (34)) describing the radial-temporal evolution of the magnetic field, contains modified Bessel functions $K_{\mu}$, which are real positive functions tending to zero at infinity, and which consistently describe the properties of the global magnetic field along the radial direction. The latitudinal distribution of $\widetilde{A}$, governed by the selfsimilar solution (Equation (29)) of the stationary state Schrödinger Equation (26), also satisfies the boundary condition (Equation (28)) for the dipole magnetic field. As for the pressure/density, the solutions (Equations (37 and 40)), respectively, are proportional to $\widetilde{A}^{2}$ which ensures their positively prescribed nature throughout the whole domain. Hence, we do not solve the initial-value problem in its usual sense, but instead find a self-similar solution that is physically acceptable for the thermodynamic variables (pressure and density) and satisfies the initial/boundary conditions for the global, dipolar magnetic field.

In our case, the self-similar theory, as a mathematical method, provides the opportunity to study a highly nonlinear process of coronal transients (CMEs) in terms of basic analytic calculations. In particular, we are able to reduce the nonlinear elliptic partial differential Equation (14), governing the fully developed MHD flow, to the set of the linear ordinary Equations (24) and (25). The solutions for magnetic field scalar potential, pressure, and density in Equations (35), (36), and (39), respectively, vary in space and time in the self-similar way and their spatial-temporal evolution is expressed via self-similar variable $\zeta=r / \Phi(t)$.

In this study, we consider the case of inertial ( $\alpha=0)$ selfsimilar flows, which is justified by observations. The velocity measurements by space-borne coronagraphs rarely show the decelerating/accelerating speeds of transient leading edges above $2 R_{0}$. Instead, observations usually show the constant speeds falling into a broad range of $100-1000 \mathrm{~km} \mathrm{~s}^{-1}$ (Gosling et al. 1976; MacQueen 1980). In other words, the inertial state is the preferred state of the transient flow and the constant upward velocities are in accordance with the assumed self-similarity (Low 1984b).

We suppose that our analytic self-similar, pressure-prescribed model can be applied to describe the evolution of real coronal transients. As a practical example, we consider the special group of observed ICMEs discussed in the paper by Jian et al. (2006). In this example, we show that our solution may successfully mimic the temporal behaviors of different ICME parameters, such as total pressure and density. More detailed calculations and free parameter fittings, which modify the self-similar solutions to adjust them to particular ICME event, will be the subject of our future works.

Finally, it is important to stress once again that the important feature of our approach that makes it original and different from that by Low (1984b) and Gibson \& Low (1998) is that our selfsimilar MHD solutions have positively defined thermal pressure. Therefore, this approach allows self-similar solutions that are well defined and physically meaningful throughout the entire domain.

The research of D.S. and A.R. was supported by the Georgian National Science Foundation grant GNSF/ST07/4-193. A.R. is grateful to Katholieke Universiteit Leuven for partial support through the Senior Research Fellow award. These results were obtained in the framework of projects GOA/2009-009 (K.U. Leuven), G.0304.07 (FWO-Vlaanderen), and C 90205 (ESA Prodex 9). Financial support by the European Commission through the SOLAIRE Network (MTRN-CT-2006-035484) is gratefully acknowledged. We also acknowledge the anonymous referee for very useful suggestions and remarks.

\section{APPENDIX}

Numerical analysis of the recurrence formula (Equation (30)) along with the boundary condition (Equation (31)) reveals the graphical relationship between $\left(\sigma, \lambda^{2}\right)$ pairs, admissible for the special solution of the problem, as shown in Figure 6. However, in order to obtain physically meaningful solutions one has to take into account the restrictions for the values of $\left(\sigma, \lambda^{2}\right)$ pairs.

In particular,

1. in order to generate the real functions of $£_{\mu}=e^{i \mu \pi} K_{\mu}$ one has to keep $\mu=0$;

2. since $\lambda^{2} \equiv 1 / 4-\mu^{2}$, the values of $\lambda^{2}$ are also restricted: $\lambda^{2}=1 / 4$

3. setting $\mu=0$ in Equation (34) restricts the function $A(\zeta)$ from above modeling the single "blob" configuration of magnetic flux rope (see Figures 1 and 2); 


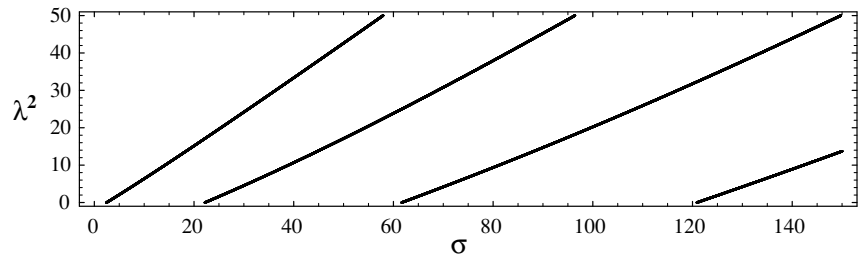

Figure 6. Contour plots of $\left(\sigma, \lambda^{2}\right)$ pairs. The first contour from the left corresponds to the "ground state" of the system. The next contours correspond to the first, second, and higher states of the system.

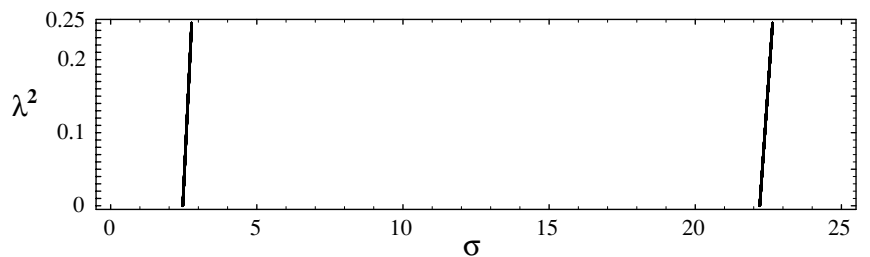

Figure 7. Zoom in plot of $\left(\sigma, \lambda^{2}\right)$ contour plots. The value of $\lambda^{2}$ is limited by $1 / 4$.

4. the zooming of the plot of $\left(\sigma, \lambda^{2}\right)$ contours, shown in Figure 7, makes clear that each specified value of $\sigma$ may pair only with one value of $\lambda^{2}$;

5. for simplicity, we model the dipolar magnetic field of the solar transient.

Therefore, we consider only those pairs of $\left(\sigma, \lambda^{2}\right)$ which are located along the first contour ("ground state" of the system) on the left-hand side of the plots in Figures 6 and Figure 7. The latitudinal distribution of the magnetic field, governed by function $T(\theta)$ (see Equation (23)) is dipolar if $\left(\sigma, \lambda^{2}\right)$ pairs originate from the "ground state" of the system. Alternatively, the magnetic field becomes multipolar if one considers $\left(\sigma, \lambda^{2}\right)$ pairs from the first, second, and higher states of the system (the next contours on the plot in Figures 6 and 7).

\section{REFERENCES}

Abramowitz, M., \& Stegun, I. 1972, in Handbook of Mathematical Functions, ed. M. Abramovitz \& I. Stegan (New York: Dover), 377

Barenblatt, G. I., \& Zel'dovich, Ya. B. 1972, ARA\&A, 4, 285

Bernstein, I. B., \& Kulsrud, R. M. 1965, ApJ, 142, 479

Burkepile, J. T., \& Cyr, O. C. St. 1993, A Revised and Expanded Catalogue of Mass Ejections Observed by the Solar Maximum Mission Coronagraph (Tech. Rep. NCAR/TN-369+STR; Boulder, CO: National Center for Atmospheric Research)

Chandrasekhar, S. 1952, Proc. Natl Acad. Sci., 42, 1

Debosscher, A. 1991, Phys. Rev. A, 44, 908

Dulk, G. A., et al. 1976, Sol. Phys., 49, 369

Finn, J. M., Lapenta, G., \& Li, H. 2004, Phys. Plasmas, 11, 2082

Forbes, T. G., et al. 2006, Space Sci. Rev., 123, 251

Gibson, S. E., \& Low, B. C. 1998, ApJ, 493, 460

Gosling, J. T., Hildner, E., MacQueen, R. M., Munro, R. H., Poland, A. I., \& Ross, C. L. 1976, Sol. Phys., 48, 389

Gosling, J. T. 1993, J. Geophys. Res., 98, 18937

Jian, L., Russel, C. T., Luhmann, J. G., \& Skoug, R. M. 2006, Sol. Phys., 239, 393

Klimchuk, J. A. 2001, in Space Weather, Geophysical Monograph 125, ed. P. Song, H. Singer, \& G. Siscoe (Washington, DC: American Geophysical Union), 143

Korobeinikov, V. P. 1956, Dokl. Akad. Nauk SSSR, 109, 271

Kulsrud, R. M., Bernstein, I. B., Kruskal, M., Fanucci, J., \& Ness, N. 1965, ApJ, 142,491

Landau, L. D., \& Lifshitz, E. M. 1981, Quantum Mechanics: Non Relativistic Theory (Vol. 3; Burlington, MA: Butterworth-Heinemann)

Low, B. C. $1982 \mathrm{a}, \mathrm{ApJ}, 254,796$

Low, B. C. 1982 b, ApJ, 261, 351

Low, B. C. $1984 \mathrm{a}$, ApJ, 281, 381

Low, B. C. 1984 b, ApJ, 281, 392

Low, B. C. 1990, ARA\&A, 28, 491

Low, B. C. 1992, ApJ, 390, 567

MacQueen, R. M. 1980, Phil. Trans. R. Soc. A, 297, 605

MacQueen, R. M., \& Fisher, R. 1983, Sol. Phys., 89, 89

Munro, R. H. 1977, BAAS, 9, 371

Sedov, L. I. 1946, Prikl. Mat. Mekh., 10, 241

Taylor, G. I. 1950, Proc. R. Soc. A, 201, 1975

Trussoni, E., \& Tsinganos, K. 1993, A\&A, 269, 589

Trussoni, E., Tsinganos, K., \& Sauty, C. 1997, A\&A, 325, 1099

Tsinganos, K. 1981, ApJ, 245, 764

Wagner, W. J., Hildner, E., House, L. L., Sawyer, C., Sheridan, K. V., \& Dulk, G. A. 1981, ApJ, 244, L123 